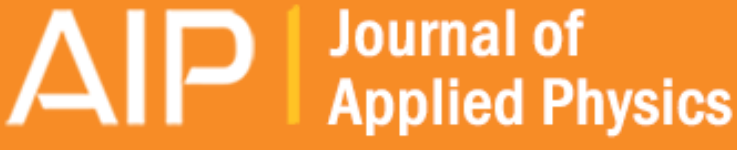

\section{Influence of a $\mathrm{Bi}$ surfactant on $\mathrm{Sb}$ incorporation in InAsSb alloys}

Evan M. Anderson, Adam M. Lundquist, Wendy L. Sarney, Stefan P. Svensson, Peter J. Carrington, Chris

Pearson, and Joanna M. Millunchick

Citation: Journal of Applied Physics 116, 014901 (2014); doi: 10.1063/1.4886635

View online: http://dx.doi.org/10.1063/1.4886635

View Table of Contents: http://scitation.aip.org/content/aip/journal/jap/116/1?ver=pdfcov

Published by the AIP Publishing

\section{Articles you may be interested in}

Metamorphic InAsSb/AllnAsSb heterostructures for optoelectronic applications

Appl. Phys. Lett. 102, 111108 (2013); 10.1063/1.4796181

Enhanced $\mathrm{Sb}$ incorporation in InAsSb nanowires grown by metalorganic vapor phase epitaxy

Appl. Phys. Lett. 98, 113104 (2011); 10.1063/1.3566980

High quality InAsSb grown on InP substrates using Al Sb/Al As Sb buffer layers

Appl. Phys. Lett. 92, 062111 (2008); 10.1063/1.2836947

Interface band gap engineering in InAsSb photodiodes

Appl. Phys. Lett. 87, 102103 (2005); 10.1063/1.2041818

Electrical Properties of InAs-Based Nanowires

AIP Conf. Proc. 723, 449 (2004); 10.1063/1.1812127

\section{AIP $\mid$ APL Photonics}

APL Photonics is pleased to announce Benjamin Eggleton as its Editor-in-Chief

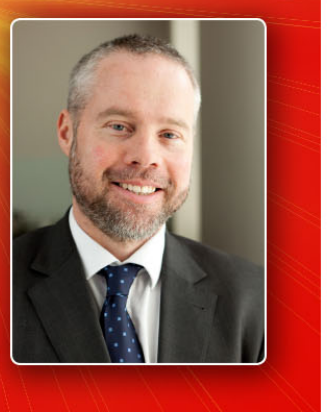




\title{
Influence of a Bi surfactant on Sb incorporation in InAsSb alloys
}

\author{
Evan M. Anderson, ${ }^{1}$ Adam M. Lundquist, ${ }^{1}$ Wendy L. Sarney, ${ }^{2}$ Stefan P. Svensson, ${ }^{2}$ \\ Peter J. Carrington, ${ }^{3}$ Chris Pearson, ${ }^{4}$ and Joanna M. Millunchick ${ }^{1}$ \\ ${ }^{1}$ Department of Materials Science and Engineering, University of Michigan, Ann Arbor, Michigan 48109, USA \\ ${ }^{2}$ Sensors \& Electron Devices Directorate, US Army Research Laboratory, Adelphi, Maryland 20783, USA \\ ${ }^{3}$ Department of Electronic and Electrical Engineering, University College London, London WCIE 7JE, \\ United Kingdom \\ ${ }^{4}$ Department of Computer Science, Engineering, and Physics, University of Michigan-Flint, Flint, \\ Michigan 48502, USA
}

(Received 6 June 2014; accepted 9 June 2014; published online 1 July 2014)

\begin{abstract}
The influence of using a Bi surfactant during the growth of InAsSb on the composition was examined, and it was found that increasing $\mathrm{Bi}$ flux on the surface during growth inhibits the incorporation of $\mathrm{Sb}$. Analysis of the data via a kinetic model of anion incorporation shows that surface Bi acts as a catalyst for InAs formation, thus inhibiting Sb incorporation. (C) 2014 AIP Publishing LLC.

[http://dx.doi.org/10.1063/1.4886635]
\end{abstract}

\section{INTRODUCTION}

InAsSb is of interest for long wavelength infrared applications because it is a direct gap III-V semiconductor that can attain sufficiently narrow band gaps, depending on the composition. ${ }^{1}$ In order to be used effectively in optoelectronic devices, the material must be grown with smooth interfaces, minimal defects, and be compositionally homogeneous. Recent work shows that fully relaxed InAsSb grown on compositionally graded buffer layers at $415^{\circ} \mathrm{C}$ is free of phase separation or ordering, even for high $\mathrm{Sb}$ compositions approaching $50 \% .{ }^{1}$ However, the optimum substrate temperature and III/V flux ratios have not been systematically investigated for these alloys. Further improvements could possibly also be obtained by the use of surfactants but their influence on the alloy composition must first be determined.

It has been suggested that the use of surfactants may improve the crystal growth of certain alloys. For instance, growing GaNAs under a Bi flux results in smoother surfaces and higher $\mathrm{N}$ incorporation. ${ }^{2}$ Similar effects were found for $\mathrm{B}$ incorporation in GaAs. ${ }^{3}$ The use of a Bi surfactant has also been observed to inhibit In segregation and decrease interfacial roughness in $\mathrm{InGaAs} / \mathrm{GaAs}{ }^{4}$ Several mechanisms for these improvements have been proposed. One suggests that surface $\mathrm{Bi}$ atoms prevent other growth processes by blocking other reactive sites. ${ }^{4}$ Another proposes that Bi displaces one anion in favor of the other. ${ }^{2}$ The effect of $\mathrm{Bi}$ on the growth of InAsSb, on the other hand, has not been reported. In this study, we investigated how the presence of a Bi flux influences the composition of InAsSb alloys grown via molecular beam epitaxy (MBE) and show that Sb incorporation is decreased with increasing Bi flux. We have developed a kinetic model that suggests that the suppression of Sb incorporation is due to a catalytic effect where $\mathrm{Bi}$ atoms on the surface are more likely to be replaced by impinging As atoms than impinging $\mathrm{Sb}$ atoms during growth.

\section{EXPERIMENTAL PROCEDURE}

InAsSb films were grown on (001) oriented GaSb substrates in two different labs. All samples were grown in
MBE chambers using solid sources for In, $\mathrm{Ga}$, and $\mathrm{Bi}$, and valved sources with cracking zones for $\mathrm{Sb}_{2}$ and $\mathrm{As}_{2}$. We grew two series at two different growth temperatures and growth rates, which are summarized in Table I. The first series (Series A) was grown at $385^{\circ} \mathrm{C}$ with $\mathrm{R}_{\mathrm{In}}=0.5 \mathrm{ML} / \mathrm{s}$, measured using reflection high energy electron diffraction (RHEED) oscillations, beam equivalent pressures (BEP) of $2.1 \times 10^{-6}$ Torr for $\mathrm{As}_{2}$, and $0.62 \times 10^{-7}$ Torr for $\mathrm{Sb}$ to a thickness of $4000 \AA$. The second series (Series B) was grown at $415^{\circ} \mathrm{C}$ with $\mathrm{R}_{\mathrm{In}}=0.9 \mathrm{ML} / \mathrm{s}, 5.7 \times 10^{-6} \mathrm{Torr}^{\mathrm{As}} 2 \mathrm{BEP}$, and $1.2 \times 10^{-7}$ Torr Sb BEP to a thickness of $2500 \AA$ or $5000 \AA$ as noted. The Bi BEP was varied up to $2.6 \times 10^{-7}$ Torr in one laboratory (series A) and $4.8 \times 10^{-7}$ Torr in the other (series B). The In, As, and Sb fluxes were calculated as described previously ${ }^{5}$ that is, by starving the growth surface of anions until we observed a decrease in the growth rate. Under these conditions, $\mathrm{V} / \mathrm{III}=1$, thus the anion flux is equal to the cation flux measured by RHEED oscillations. Alternatively, the anion incorporation rate can be determined using anion uptake oscillations, ${ }^{6}$ which we find correlate well to the anion starvation method. Bi fluxes could not be calculated using this approach because RHEED uptake oscillations were not observable, nor is the transition from anion to cation terminated surfaces known. Compositional analysis was conducted using high resolution x-ray diffraction (XRD) and Rutherford Backscattering Spectrometry (RBS). The compositions were calculated for the samples using two (004) scans with a $180^{\circ}$ rotation in phi and two (115) scans with a $180^{\circ}$ rotation in phi and taken in glancing incidence and exit. SimNRA software ${ }^{7}$ was used to analyze the RBS data.

\section{THE MODEL}

There are several kinetic models for mixed anion alloy growth, ${ }^{5,8-10}$ each of which takes the various physical processes that take place into account. For instance, the incident flux is the primary term that determines the net growth flux, but this contribution is influenced by the presence of desorption and surface segregation. Another process known to 
TABLE I. Growth conditions for InAsSb films grown on GaSb substrates.

\begin{tabular}{|c|c|c|c|c|c|c|}
\hline & $\mathrm{T}\left({ }^{\circ} \mathrm{C}\right)$ & $\mathrm{F}_{\mathrm{In}}\left(\times 10^{14} \mathrm{~cm}^{-2} \mathrm{~s}^{-1}\right)$ & $\mathrm{F}_{\mathrm{As} 2}\left(\times 10^{14} \mathrm{~cm}^{-2} \mathrm{~s}^{-1}\right)$ & $\mathrm{F}_{\mathrm{Sb}}\left(\times 10^{14} \mathrm{~cm}^{-2} \mathrm{~s}^{-1}\right)$ & $\operatorname{Bi} \operatorname{BEP}\left(\times 10^{-7}\right.$ Torr $)$ & $h(\AA)$ \\
\hline \multirow[t]{4}{*}{ Series A } & 385 & 2.8 & 7.1 & 0.8 & 0.0 & 4000 \\
\hline & & 2.8 & 7.1 & 0.8 & 0.7 & 4000 \\
\hline & & 2.8 & 7.1 & 0.8 & 1.2 & 4000 \\
\hline & & 2.8 & 7.1 & 0.8 & 2.8 & 4000 \\
\hline \multirow[t]{4}{*}{ Series B } & 415 & 5.0 & 15.1 & 1.4 & 0.0 & 2500 \\
\hline & & 5.0 & 15.1 & 1.4 & 1.0 & 5000 \\
\hline & & 5.0 & 15.1 & 1.4 & 2.6 & 5000 \\
\hline & & 5.0 & 15.1 & 1.4 & 4.8 & 5000 \\
\hline
\end{tabular}

occur in mixed anion systems is the removal of one anion species in place of another. ${ }^{11}$ For instance, it is well known that As preferentially displaces $\mathrm{Sb}$ in both $\mathrm{GaAsSb}^{11}$ and InAsSb. ${ }^{5}$ Each of these rates is Arrhenius in nature and takes the standard form

$$
P=\frac{(N \nu)^{2}}{F_{I n}} \exp \left[-\frac{2 E_{j}}{k T}\right],
$$

where $\mathrm{N}$ is the density of atomic surface sites, $\nu$ is the attempt frequency, $E_{j}$ is the activation energy of the $j$ th process, $\mathrm{k}$ is Boltzmann's constant, and $\mathrm{T}$ is the temperature. $\mathrm{F}_{\mathrm{In}}$ is the impinging In flux, which acts to limit the process.

To date, these models have only taken binary alloys into account, but it is straightforward to add a third anion atom to the model. The growth flux for $\mathrm{Bi}$ is as follows:

$$
\begin{aligned}
J_{B i}= & F_{B i}-\theta_{B i} P_{B i}^{d e s}-\theta_{B i} P_{B i \rightarrow A s}^{r e m}-\theta_{B i} P_{B i \rightarrow S b}^{r e m} \\
& +y_{b} \theta_{A s} P_{A s \rightarrow B i}^{s e g}+y_{b} \theta_{S b} P_{S b \rightarrow B i}^{s e g} .
\end{aligned}
$$

The first term on the right hand side represents the amount of impinging $\mathrm{Bi}, \mathrm{F}_{\mathrm{Bi}}$. The second term represents the reduction in the net flux due to desorption, which is the product of the rate of desorption and the surface coverage of $\mathrm{Bi}, \theta_{\mathrm{Bi}}$. The third and fourth terms decrease the net flux of $\mathrm{Bi}$ via removal of $\mathrm{Bi}$ from the surface by $\mathrm{As}$ or $\mathrm{Sb}$, and depend on the product of the coverage of $\mathrm{Bi}$ and the rate of removal. The fifth and sixth terms increase the net flux of $\mathrm{Bi}$ due to surface segregation, the exchange of subsurface $\mathrm{Bi}$ and $\mathrm{As}$ or $\mathrm{Sb}$ on the surface. These terms are the product of the composition of $\mathrm{Bi}$ in the subsurface layer (assumed to be the same as that of the bulk) $\mathrm{y}_{\mathrm{b}}$, the surface coverage of $\mathrm{As}$ or $\mathrm{Sb}$, and the rate of segregation. $\mathrm{y}_{\mathrm{b}}$ is proportional to the surface coverage of $\mathrm{Bi}$ via the equilibrium partition coefficient $\mathrm{K}$, and can range from 0 (for a pure surfactant) to 1 (for complete incorporation). ${ }^{12}$ Similar equations can be written for the growth flux of $\mathrm{Sb}$ and $\mathrm{As}$

$$
\begin{aligned}
J_{S b}= & F_{S b}-\theta_{S b} P_{S b}^{d e s}-\theta_{S b} P_{S b \rightarrow A s}^{r e m}+\theta_{B i} P_{B i \rightarrow S b}^{r e m} \\
& +x_{b} \theta_{A s} P_{A s \rightarrow S b}^{s e g}-y_{b} \theta_{S b} P_{S b \rightarrow B i}^{s e g}, \\
J_{A s}= & F_{A s}-\theta_{A s} P_{A s}^{d e s}+\theta_{S b} P_{S b \rightarrow A s}^{r e m}+\theta_{B i} P_{B i \rightarrow A s}^{r e m} \\
& -x_{b} \theta_{A s} P_{A s \rightarrow S b}^{s e g}-y_{b} \theta_{A s} P_{A s \rightarrow B i}^{s e g} .
\end{aligned}
$$

The concentration of each anion is thus the ratio of the net flux of that anion and the sum of the net anion fluxes.

\section{EXPERIMENTAL RESULTS AND DISCUSSION}

Figure 1 shows (004) XRD reciprocal space maps (RSMs) of series A under various Bi fluxes. The substrate and film peaks are easily discernible in each map. The clearest trend from these maps is that the amount of $\mathrm{Sb}$ incorporated decreases with increasing Bi flux, as shown by the increasing film-substrate splitting. The film peak for the film grown without a Bi flux is diffused and elongated along the vertical direction, suggesting an out of plane lattice distortion, possibly due to variations in composition. This peak is also broadened indicating lateral variation in the lattice parameter possibly due to defect injection and strain relaxation (10\%) arising from the lattice mismatch between the film and substrate. In contrast, the films grown under a Bi flux exhibit diffraction peaks that are narrow and isotropic, suggesting high crystalline quality and uniformity in composition. The sample grown under the highest Bi flux again shows some peak broadening, most likely due to dislocations arising from the increasing lattice mismatch. The relaxation in this sample was $4 \%$.

Figure 2 shows plots of the $\mathrm{Sb}$ composition determined from the XRD measurements as a function of Bi BEP for the two series. Given that $\mathrm{Bi}$ is known to readily desorb at these temperatures, ${ }^{13}$ it was assumed that no $\mathrm{Bi}$ was incorporated into the films when calculating the compositions. It is clear that increased $\mathrm{Bi}$ flux decreases the amount of $\mathrm{Sb}$ incorporation for constant growth conditions. As shown in Eqs. (2)-(4), the net growth rates of each species depends on a number of factors, including desorption, surface segregation, and anion exchange. Furthermore, the presence of $\mathrm{Bi}$ on the surface is expected to alter these processes. The influence of $\mathrm{Bi}$ on the composition appears to be stronger for series $\mathrm{A}$ (low T) than series B (high T), which may be due to the difference in growth temperatures. However, there may also be a difference in the absolute $\mathrm{Bi}$ fluxes between the two sample series, as ion gauge sensitivity factors are known to vary depending on the gauge geometry and history. ${ }^{14}$

RBS experiments were performed to confirm the assumption that no $\mathrm{Bi}$ had incorporated into the films. A beam of $1.9 \mathrm{MeV} \mathrm{He}{ }^{++}$ions incident normal to the sample surface was used with a $20^{\circ}$ detector angle. Figure 3 shows the RBS spectra for series A along with the simulated curve and the elemental components for the sample grown without Bi. The vertical dotted line denotes the channel at which the $\mathrm{Bi}$ signal would be observed and confirms that there is no $\mathrm{Bi}$ in the films greater than the detection limit of the RBS $(0.5$ atomic \%). The RBS for these films shows the plateaus 
(a)
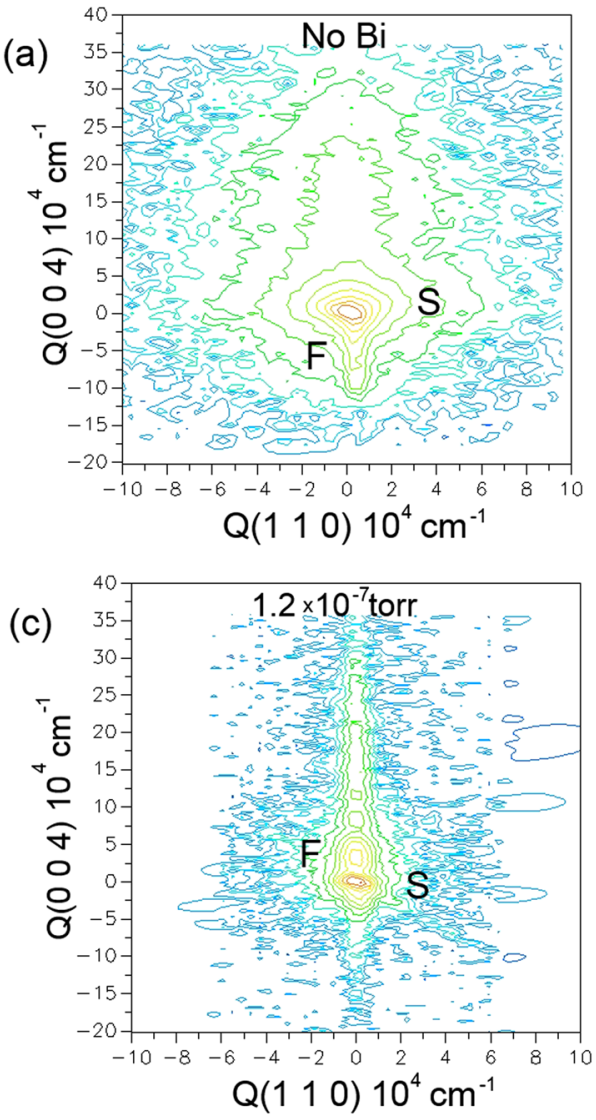

(b)

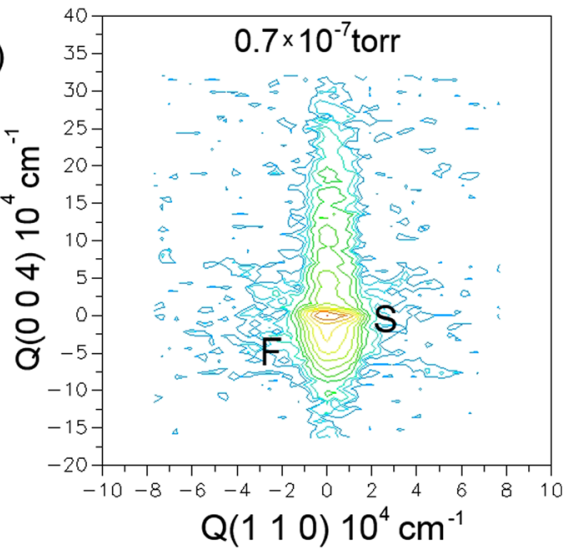

(d)

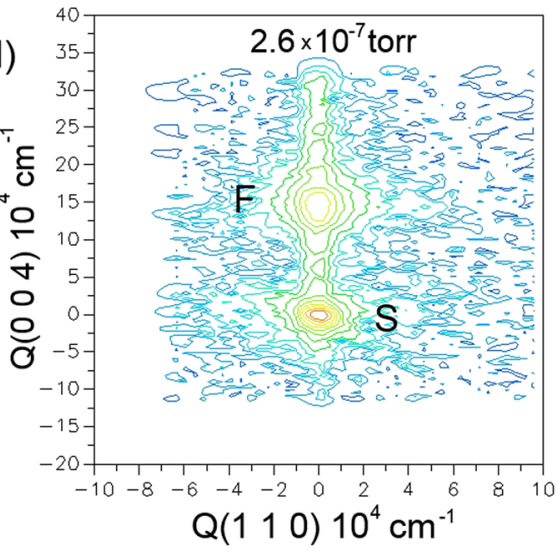

FIG. 1. (004) Reciprocal space maps of series A films. S denotes the GaSb substrate and $\mathrm{F}$ denotes the InAsSb film.

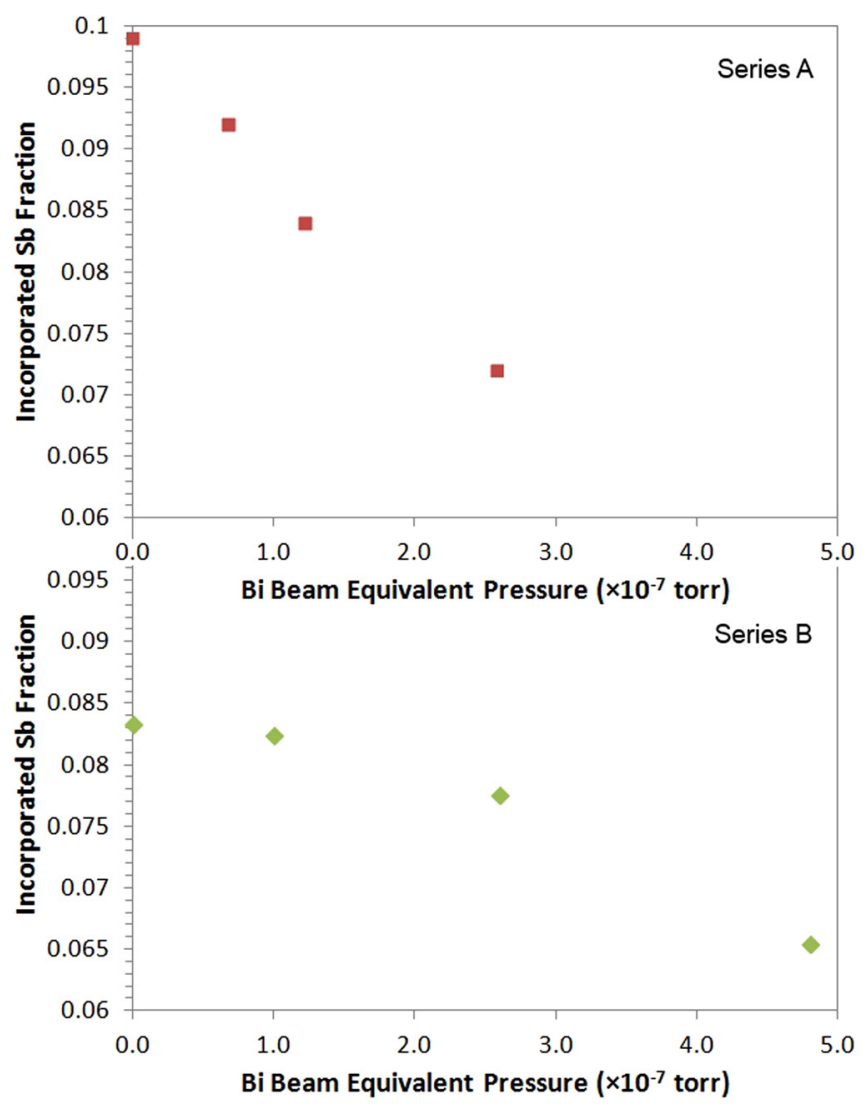

FIG. 2. Plots of film composition vs Bi flux for series A and series B. that would be expected for homogeneous films, whose positions are consistent with the composition determined by XRD. The peaks and valleys in the RBS for the films grown with $\mathrm{Bi}$ arise due to the superposition of the signals from each element in the layers assuming abrupt interfaces. This is consistent with the XRD data of these films, which exhibit Pendellosung fringes (Figs. 1(b)-1(d)). The RBS of the film grown without $\mathrm{Bi}$ lacks these distinct peaks, suggesting a rough interface between the InAsSb film and the $\mathrm{GaSb}$ substrate. This observation is also consistent with the lattice-mismatch induced broadening observed in the XRD

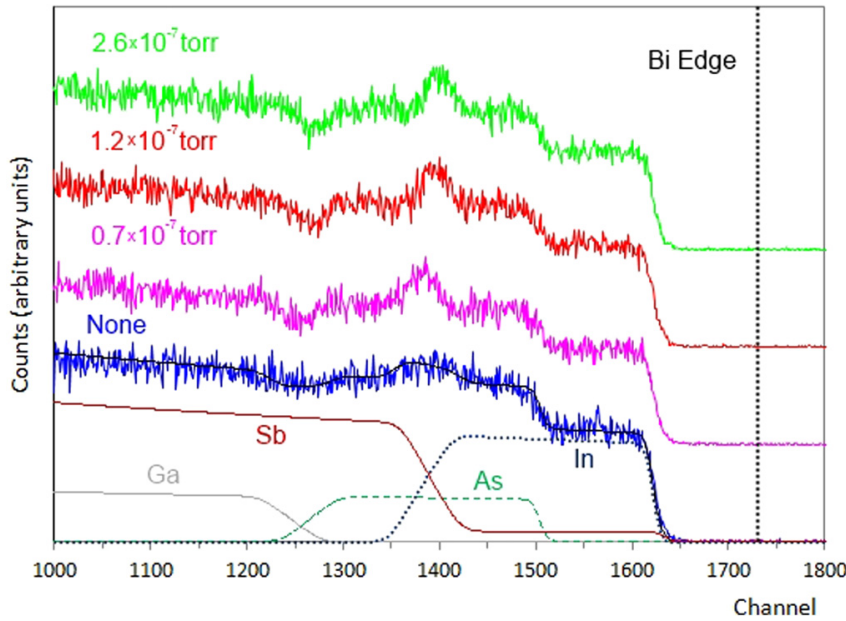

FIG. 3. Plots of RBS spectra of series A as a function of Bi BEP. The simulated curve and its elemental components are included for the samples grown without $\mathrm{Bi}$. The channel at which $\mathrm{Bi}$ would be observed is marked by a dotted line. 
for this film (Fig. 1(a)). The RBS spectra of series B had no evidence of $\mathrm{Bi}$ incorporation either.

These experimental results show that some of the processes that were identified in Eqs. (2) through (4) can be eliminated. For instance, the RBS data show no Bi incorporation into the InAsSb film for both series. Thus $\mathrm{y}_{\mathrm{b}}$ and $\mathrm{J}_{\mathrm{Bi}}$ are zero. As a result, the impinging flux of $\mathrm{Bi}$ is equal to the sum of the desorption and removal fluxes such that

$$
F_{B i}-\theta_{B i} P_{B i}^{d e s}=\theta_{B i} P_{B i \rightarrow A s}^{r e m}+\theta_{B i} P_{B i \rightarrow S b}^{r e m} .
$$

Given that both $\mathrm{J}_{\mathrm{Sb}}$ and $\mathrm{J}_{\mathrm{As}}$ depend on the removal of $\mathrm{Bi}$ by that anion, Eq. (5) may substitute for the fourth terms in Eqs. (3) and (4). The concentration of $\mathrm{Sb}$ is the net $\mathrm{Sb}$ flux divided by the sum of all of the net anion fluxes. Since the only growth parameter that was varied in each series is the flux of $\mathrm{Bi}$, it is reasonable to assume that the desorption, removal, and segregation terms in each net flux equation are constant. If in this case, $\mathrm{Bi}$ removal by $\mathrm{Sb}$ is much faster than or equal to $\mathrm{Bi}$ removal by $\mathrm{As}$, the $\mathrm{Sb}$ concentration will increase with increasing $\mathrm{Bi}$ flux. If instead $\mathrm{Bi}$ removal by $\mathrm{As}$ is faster than the removal of $\mathrm{Bi}$ by $\mathrm{Sb}$, then the $\mathrm{Sb}$ concentration will decrease with increasing Bi flux. The experimental results show that $\mathrm{Sb}$ incorporation decreases with increasing Bi flux, therefore the latter case is more likely.

These experiments show that surface Bi acts as a catalyst for As incorporation. This is not surprising considering that the In-As bond is stronger than the In-Sb or (calculated) In-Bi bonds. ${ }^{15}$ This catalytic model is consistent with the results for GaNAs, which showed enhanced $\mathrm{N}$ incorporation in the presence of $\mathrm{Bi}^{2}$ In that case, the $\mathrm{Ga}-\mathrm{N}$ bond is stronger than the Ga-As or (calculated) $\mathrm{Ga}-\mathrm{Bi}$ bonds, ${ }^{15}$ resulting in faster removal of Bi by $\mathrm{N}$ than by As. These results together demonstrate the importance of the surfactant in changing the chemistry of the surface, rather than acting as a steric barrier to incorporation.

\section{CONCLUSIONS}

Experimental evidence shows that the presence of $\mathrm{Bi}$ as a surfactant inhibits $\mathrm{Sb}$ incorporation in InAsSb alloys for a range of growth conditions without itself incorporating. A proposed kinetic model suggests that surface $\mathrm{Bi}$ atoms increase the reactivity of As on the surface. Further study is required to systematically quantify all the mechanisms involved, but this model can be used to determine the composition function of $\mathrm{Bi}$ flux, and be extended to describe other mixed anion or mixed/cation systems, such as GaAsN or AlInAsSb.

\section{ACKNOWLEDGMENTS}

E.M.A., A.M.L., C.P., and J.M.M. gratefully acknowledge Chakrapani Varanasi and the support of the Department of Defense, Army Research Office via the Grant No. W911NF-12-1-0338.

${ }^{1}$ S. P. Svensson, W. L. Sarney, H. Hier, Y. Lin, D. Wang, D. Donetsky, L. Shterengas, G. Kipshidze, and G. Belenky, Phys. Rev. B 86, 245205 (2012).

${ }^{2}$ E. C. Young, S. Tixier, and T. Tiedje, J. Cryst. Growth 279, 316 (2005).

${ }^{3}$ A. J. Ptak, D. A. Beaton, and A. Mascarenhas, J. Cryst. Growth 351, 122 (2012).

${ }^{4}$ M. Pillai, S. Kim, S. Ho, and S. Barnett, J. Vac. Sci. Technol., B 18, 1232 (2000).

${ }^{5}$ J. M. Millunchick, E. M. Anderson, C. Pearson, W. L. Sarney, and S. P. Svensson, J. Appl. Phys. 114, 234907 (2013).

${ }^{6}$ B. F. Lewis, R. Fernandez, A. Madhukar, and F. J. Grunthaner, J. Vac. Sci. Technol., B 4, 560 (1986).

${ }^{7}$ M. Mayer, in The Fifteenth International Conference on the Application of Accelerators in Research and Industry, Denton, TX,4-7 Nov. 1998 (AIP, 1999), pp. 541-544.

${ }^{8}$ B. W. Liang and C. W. Tu, J. Appl. Phys. 74, 255 (1993).

${ }^{9}$ J.-M. Lin, L.-C. Chou, and H.-H. Lin, J. Vac. Sci. Technol., B 29, 021011 (2011).

${ }^{10}$ X. Lu, D. Beaton, R. Lewis, T. Tiedje, and M. Whitwick, Appl. Phys. Lett. 92, 192110 (2008).

${ }^{11}$ M. Losurdo, P. Capezzuto, G. Bruno, A. S. Brown, T. Brown, and G. May, J. Appl. Phys. 100, 013531 (2006).

${ }^{12}$ J. Y. Tsao, Materials Fundamentals of Molecular Beam Epitaxy (Academic Press, Boston, 1993).

${ }^{13}$ E. C. Young, M. B. Whitwick, T. Tiedje, and D. A. Beaton, Phys. Status Solidi C 4, 1707 (2007).

${ }^{14}$ T. A. Flaim and P. D. Ownby, J. Vac. Sci. Technol. 8, 661 (1971).

${ }^{15}$ M. A. Berding, A. Sher, A. X. B. Chen, and W. E. Miller, J. Appl. Phys. 63, 107 (1988). 